Article

\title{
Investigations on the Cosputtered ITO-ZnO Transparent Electrode Ohmic Contacts to $n-\mathrm{GaN}$
}

\author{
Wei-Hua Hsiao ${ }^{1}$, Tai-Hong Chen ${ }^{2}$, Li-Wen Lai ${ }^{2}$, Ching-Ting Lee ${ }^{3}$, Jyun-Yong Li $^{1}$, \\ Hong-Jyun Lin ${ }^{1}$, Nan-Jay Wu ${ }^{1}$ and Day-Shan Liu ${ }^{1, *}$ \\ 1 Institute of Electro-Optical and Materials Science, National Formosa University, Yunlin 632, Taiwan; \\ s706802000213@gmail.com (W.-H.H.); 10476110@gm.nfu.edu.tw (J.-Y.L.); n125343@yahoo.com.tw (H.-J.L.); \\ tsaicc1221@gmail.com (N.-J.W.) \\ 2 Industrial Technology Research Institute South, Tainan 734, Taiwan; a0922639175@gmail.com (T.-H.C.); \\ ting3561387@yahoo.com.tw (L.-W.L.) \\ 3 Institute of Microelectronics, Department of Electrical Engineering, National Cheng Kung University, \\ Tainan 701, Taiwan; tsaicc@ee.ncku.edu.tw \\ * Correspondence: dsliu@nfu.edu.tw; Tel.: +886-5-6315665
}

Academic Editors: Chien-Hung Liu and Huei-Chu Weng

Received: 29 December 2015; Accepted: 16 February 2016; Published: 22 February 2016

\begin{abstract}
Transparent indium tin oxide (ITO) and cosputtered ITO-zinc oxide (ZnO) films' contacts to an $n$-GaN epilayer were investigated. Both of these electrodes' contact to the $n$-GaN epilayer showed Schottky behavior, although the contact resistance of the ITO-ZnO $/ n-\mathrm{GaN}$ system was lower than that of the ITO $/ n-\mathrm{GaN}$ system. By placing a thin Ti interlayer between the ITO-ZnO/n-GaN interface, nonalloyed ohmic contact was achieved. The inset Ti interlayer was both beneficial both for enhancing the outdiffusion of the nitrogen atoms at the surface of the $n$-GaN and suppressing the indiffusion of oxygen atoms from the surface of the ITO- $\mathrm{ZnO}$ to $n$-GaN. The figure-of-merit (FOM), evaluated from the specific contact resistance and optical property of the Ti/ITO-ZnO system's contact to the $n$-GaN epilayer, was optimized further at an adequate thickness of the Ti interlayer.
\end{abstract}

Keywords: cosputtered ITO-ZnO; n-GaN; transparent electrode; Ti interlayer; ohmic contact; figure-of-merit

\section{Introduction}

Group III-nitride semiconductors are crucial materials for the fabrication of optoelectronic devices [1,2]. Among them, gallium nitride $(\mathrm{GaN})$ has been the most attractive material during the past decades due to its mature fabrication using epitaxial and doping technologies. One of the important requirements for developing high-quality, GaN-based optoelectronic devices is the formation of ohmic contact with low specific contact resistance and/or Schottky contact with quality rectifying behavior. In regard to the material's contact to GaN, opaque or semitransparent single, bilayer, and multi-metal structural layers have been used extensively as ohmic contact electrodes in contact with $\mathrm{GaN}$. Moreover, since the $n$-GaN epilayer has the advantage of a high carrier concentration, which is beneficial for current flow tunneling the space charge region, and a low work function of about $4.1 \mathrm{eV}$, many common metals, such as $\mathrm{Ti}, \mathrm{Al}, \mathrm{In}, \mathrm{Cr}$, and their combined structures have been reported to be good contact electrodes [3-5]. However, although a very low contact resistance was achieved from the above-mentioned electrodes, the resulting optoelectronic devices have very low external quantum efficiency because most of the light that is transmitted is blocked by these electrodes. Accordingly, the development of a transparent conductive oxide (TCO) film for ohmic contact to the $n$-GaN was essential to improve the performance of the device by enhancing the optical transmittance as well 
as spreading the current transmission. To date, only a few papers have reported the preparation of the TCO electrode contact to $n-\mathrm{GaN}$ and the structures showed ohmic contact behavior only through an additional process on the contact's structure or using a highly-doped $n$-GaN epilayer [6-9]. In addition, some researchers have used a thin $\mathrm{Ti}$ or In interlayer inset between the $\mathrm{TCO} / n-\mathrm{GaN}$ interface to provide a nonalloyed ohmic contact system [10,11]. For example, Sheu et al. reported that ohmic contact behavior with a specific contact resistance of $5.1 \times 10^{-4} \Omega \cdot \mathrm{cm}^{2}$ was only obtained when the ITO contacted a highly doped $n$-GaN $\left(n=1 \times 10^{19} \mathrm{~cm}^{-3}\right)$ [6]. Tang et al. demonstrated an ITO/ZnO/n-GaN ohmic contact system with a specific contact resistance of $3.0 \times 10^{-4} \Omega \cdot \mathrm{cm}^{2}$ after annealing at $500{ }^{\circ} \mathrm{C}$ in $\mathrm{H}_{2}$ ambient for $5 \mathrm{~min}$ [7]. Hwang et al. proposed a nonalloyed Ti/ITO system ohmic contact to a plasma treatment $n-\mathrm{GaN}$ surface with a specific contact resistance of $3.2 \times 10^{-6} \Omega \cdot \mathrm{cm}^{2}[8]$. Kang et al. used a thin In interlayer inset between the ITO/N-face $n$-GaN interface to realize a specific contact resistance of $1.8 \times 10^{-5} \Omega \cdot \mathrm{cm}^{2}$ after annealing at $300{ }^{\circ} \mathrm{C}$ in $\mathrm{N}_{2}$ ambient for $60 \mathrm{~s}$ [10]. Guo et al. used a thin Ti interlayer to optimize the ITO $/ n^{+}-\mathrm{GaN}$ ohmic contact behavior with a specific contact resistance of $4.2 \times 10^{-6} \Omega \cdot \mathrm{cm}^{2}$ after annealing at $600{ }^{\circ} \mathrm{C}$ in $\mathrm{N}_{2}$ ambient for 5 min [11].

In this work, a cosputtered indium tin oxide (ITO)-zinc oxide $(\mathrm{ZnO})$ film, which had a much lower resistivity than the ITO film (as described elsewhere [12]), was deposited onto the $n$-GaN epilayer. In order to produce the nonalloyed ohmic contact system, a thin interlayer of Ti was inset between the cosputtered ITO-ZnO film and the $n$-GaN epilayer. The thickness of the Ti interlayer in the Ti/ITO-ZnO system affected the optical transmittance, and the resulting specific contact resistance was systematically investigated. An optimal ITO-ZnO/Ti/n-GaN contact structure was obtained by considering both the behavior of the ohmic contact and the optical transmittance.

\section{Experimental}

A 300-nm-thick ITO film and cosputtered ITO-ZnO film were respectively deposited onto the Si-doped $n$-GaN epilayer using a radio-frequency (rf) magnetron cosputtering system. The $n$-GaN epilayer with a thickness of $2 \mu \mathrm{m}$ was grown after the growth of an undoped GaN buffer layer on $c$-plane sapphire using the metal organic chemical vapor deposition technology. Patterns for the current-voltage (I-V) measurements using the transmission-line method (TLM) were prepared through the standard photolithography and lift-off technology. ITO target was employed as the source for the ITO film deposition, while an additive $\mathrm{ZnO}$ target cosputtered with the ITO target was used to prepare the cosputtered ITO-ZnO film at an theoretical atomic ratio of $33 \%(\mathrm{Zn} /(\mathrm{Zn}+\mathrm{In})$ at. \%). The detailed deposition parameters for preparing the cosputtered ITO-ZnO film are reported elsewhere [12]. These $\mathrm{ITO} / n-\mathrm{GaN}$ and ITO-ZnO $/ n-\mathrm{GaN}$ contact systems were then rapidly annealed at 100,200 , and $300{ }^{\circ} \mathrm{C}$, respectively, for $1 \mathrm{~min}$ under vacuum ambient. Another set of the $n$-GaN epilayer surface was deposited by a Ti interlayer with the thickness ranging from $2 \mathrm{~nm}$ to $10 \mathrm{~nm}$ using the electron beam evaporation system prior to the cosputtered ITO-ZnO film deposition. The metallic Ti $(20 \mathrm{~nm}) / \mathrm{Al}(200 \mathrm{~nm})$ system prepared by using the electron beam evaporation was also deposited onto $n$-GaN epilayer as the controlled sample.

Film thicknesses were measured using a surface profile system (Dektak 6M, Veeco, New York, NY, USA). Resistivity, carrier concentration, and Hall mobility of the ITO and cosputtered ITO-ZnO films as well as the $n$-GaN epilayer were conducted using the van der Pauw method (Ecopia HMS-5000, Ecopia Anyang, Korea) at room temperature. Optical transmittance of the ITO and cosputtered ITO-ZnO film as well as the Ti/ITO-ZnO structures was measured by a UV-VIS spectrophotometer (Labomed UVD-3500, Labomed, Inc. Los Angeles, CA, USA). Auger electron spectroscopy (AES) depth profiles of the $\mathrm{Ti} / \mathrm{Al}$, Ti/ITO-ZnO structures and the ITO-ZnO film directly contact to $n$ - $\mathrm{ZnO}$ film were performed on a scanning Auger nanoprobe (Ulvac-PHI, PHI 700, ULVAC, Kanagawa, Japan). The $I-V$ characteristics of these contact systems were measured by a semiconductor parameter analyzer (HP4156C, Aglient, Santa Clara, CA, USA). 


\section{Results and Discussions}

Table 1 provides the electrical properties of the ITO and cosputtered ITO-ZnO films as well as the $n$-GaN epilayer examined from the Hall-effect measurements at room temperature. Both the ITO and cosputtered ITO-ZnO films were $n$-type degenerated semiconductors with high electron concentrations of $2.59 \times 10^{20} \mathrm{~cm}^{-3}$ and $1.54 \times 10^{21} \mathrm{~cm}^{-3}$, respectively. In company with the carrier mobility of the cosputtered ITO-ZnO film $\left(10.6 \mathrm{~cm}^{2} \mathrm{~V}^{-1} \mathrm{~s}^{-1}\right)$, which was about two-fold greater than that of the ITO film $\left(5.4 \mathrm{~cm}^{2} \mathrm{~V}^{-1} \mathrm{~s}^{-1}\right)$, the cosputtered ITO-ZnO film $\left(\sim 3.82 \times 10^{-4} \Omega \cdot \mathrm{cm}\right)$ possessed a much lower resistivity than the ITO film $\left(\sim 2.46 \times 10^{-3} \Omega \cdot \mathrm{cm}\right)$. Figure 1 shows the optical transmittance spectra of the ITO and cosputtered ITO-ZnO films. The incorporation of the $\mathrm{Zn}$ atoms into the ITO film led to the apparent red-shift of the absorption edge in the near UV wavelengths, and the optical energy band gap of the cosputtered ITO-ZnO film was decreased to $3.41 \mathrm{eV}$, as evaluated from the curves of the $(\alpha h v)^{2}$ versus the photon energy $(\mathrm{eV})$ shown in the inset figure [13]. Although the absorption onset of the cosputtered ITO-ZnO film occurred at a longer wavelength, it still had good transparency at the visible wavelengths, with an average transmittance at wavelengths of $400-700 \mathrm{~nm}$ of about $84.6 \%$ (Table 1), even a little better than that of the ITO film ( $81.1 \%)$.

Table 1. Electrical properties and average optical transmittance of the ITO and cosputtered ITO-ZnO films as well as the electrical properties of the $n$-gallium nitride $(\mathrm{GaN})$ epilayer.

\begin{tabular}{ccccc}
\hline Sample & $\boldsymbol{n}\left(\mathbf{c m}^{-3}\right)$ & $\mu_{\mathbf{n}}\left(\mathbf{c m}^{2} / \mathrm{V}_{\mathbf{S}}\right)$ & $\rho(\Omega \cdot \mathbf{c m})$ & $\boldsymbol{T}_{\text {ave }} \mathbf{( \% )}$ \\
\hline ITO & $2.59 \times 10^{20}$ & 5.4 & $2.46 \times 10^{-3}$ & 81.1 \\
ITO-ZnO & $1.54 \times 10^{21}$ & 10.6 & $3.82 \times 10^{-4}$ & 84.6 \\
$n$-GaN & $4.59 \times 10^{18}$ & 156.1 & $8.71 \times 10^{-3}$ & - \\
\hline
\end{tabular}

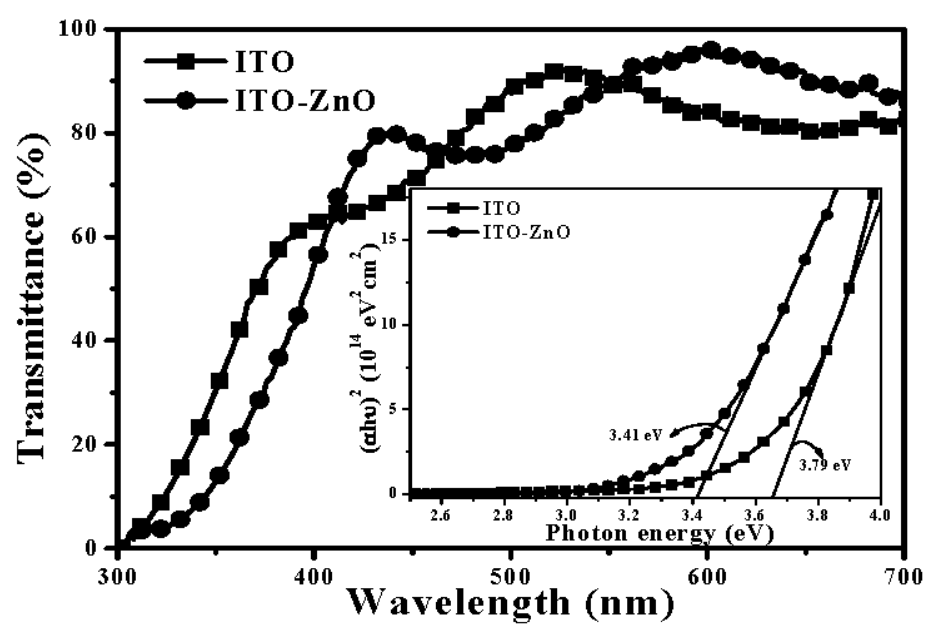

Figure 1. Optical transmittance spectra of the ITO and cosputtered ITO-ZnO films (the corresponded optical energy band gaps determined from the $(\alpha h v)^{2}$ versus the photon energy $(\mathrm{eV})$ also are provided in the inset figure).

The $I-V$ curves of the as-deposited ITO and cosputtered ITO-ZnO films' contact to the $n$-GaN epilayer, respectively, measured from the contact spacing of $15 \mu \mathrm{m}$ are shown in Figure 2. Both of these contact systems exhibited nonlinear characteristics even though the cosputtered ITO-ZnO film's contact to the $n$-GaN epilayer had a lower series resistance at an applied voltage of $2 \mathrm{~V}(\sim 485 \Omega)$ than the ITO $/ n$-GaN contact system $(\sim 784 \Omega)$. The rectifying properties with significant leakage currents measured from these contact systems occurred because of the large discrepancy in work functions between the transparent electrode and the $n$-GaN epilayer [14]. A post-annealing treatment was conducted on these contact systems for the purpose of making these structures become ohmic contacts. Figure $3 \mathrm{a}, \mathrm{b}$, respectively, give the $I-V$ curves of ITO $/ n-\mathrm{GaN}$ and ITO- $\mathrm{ZnO} / n-\mathrm{GaN}$ contact 
systems processed by a rapid thermal annealing (RTA) treatment at various temperatures for $1 \mathrm{~min}$ under vacuum ambient, measured from the contact spacing of $15 \mu \mathrm{m}$. The currents of both of these contact systems were apparently increased as the annealing temperature was increased. The largest current at a bias of $2 \mathrm{~V}$ was obtainable from the $200{ }^{\circ} \mathrm{C}$-annealed ITO/ $n$-GaN contact system, while that of the ITO- $\mathrm{ZnO} / n-\mathrm{GaN}$ contact system occurred at an annealing temperature of $300^{\circ} \mathrm{C}$. However, although the series resistance of the $300{ }^{\circ} \mathrm{C}$-annealed ITO- $\mathrm{ZnO} / n$-GaN contact system was decreased significantly to about $200 \Omega$ at a bias of $2 \mathrm{~V}$, its $I-V$ curve still was somewhat insufficient for achieving ohmic contact. In contrast to the ITO-ZnO/n-GaN contact system, the as-deposited metallic Ti/Al contact to the $n-G a N$ epilayer exhibited a good, linear $I-V$ characteristic without the requirement of a post-annealing process, as shown in Figure 4a. The specific contact resistance derived from the resistance of the $\mathrm{Al} / \mathrm{Ti} / n-\mathrm{GaN}$ contact system as a function of the contact spacing in the set figure was approximately $5.1 \times 10^{-5} \Omega \cdot \mathrm{cm}^{2}$. The mechanism responsible for the achievement of the nonalloyed ohmic contact behavior using the metallic $\mathrm{Ti} / \mathrm{Al}$ structure mainly was due to the elemental $\mathrm{Ti}$, which possessed a much lower work function $(\phi=4.3 \mathrm{eV})$. This value was only a little higher than the work function of $n-\mathrm{GaN}(\phi=4.1 \mathrm{eV})$ as compared to that of the transparent ITO $(\phi=5.1 \mathrm{eV})[15,16]$. In addition, since the electron carriers correlated with the nitrogen vacancies $\left(V_{N}\right)$ at the $n$-GaN surface would be increased as a consequence of the Ti atom, Ti was beneficial for inducing the outdiffusion of the nitrogen atoms at the $n$-GaN surface. Figure $4 \mathrm{~b}$ shows the elemental distributions over the $\mathrm{Ti} / n-\mathrm{GaN}$ interface conducted from the AES depth profile. The outdiffusion of the $\mathrm{N}$ atoms was observed clearly at the $\mathrm{Ti} / n$-GaN interface, resulting in the increase in the electron concentration at the $n$-GaN surface associated with large amounts of the $\mathrm{V}_{\mathrm{N}}$ donors $[17,18]$.

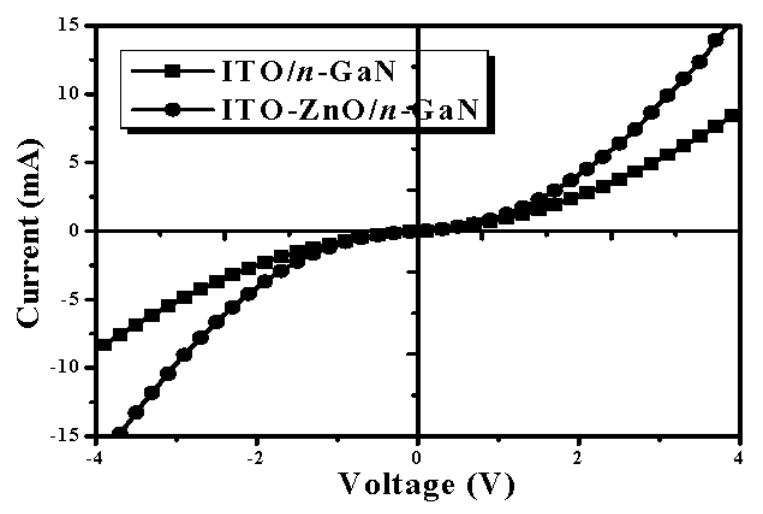

Figure 2. I- $V$ curves of the as-deposited ITO and cosputtered ITO-ZnO films' contact to $n$-GaN epilayer, respectively, measured from the contact spacing of $15 \mu \mathrm{m}$.
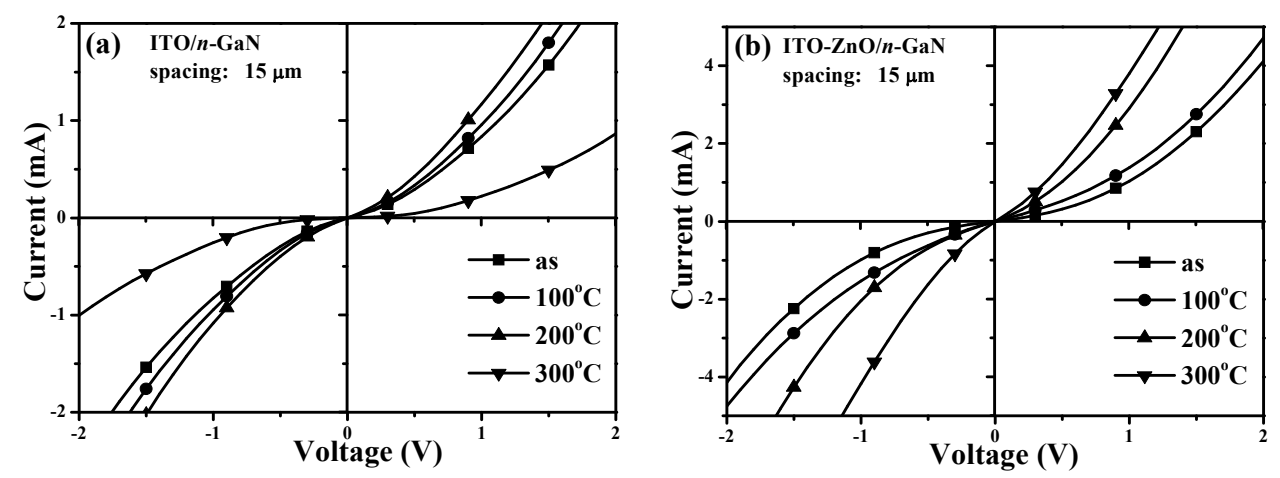

Figure 3. $I-V$ curves of the (a) ITO $/ n-\mathrm{GaN}$ and (b) ITO-ZnO/n-GaN contact systems processed by various annealing temperatures for $1 \mathrm{~min}$ under vacuum ambient, measured from the contact spacing of $15 \mu \mathrm{m}$. 

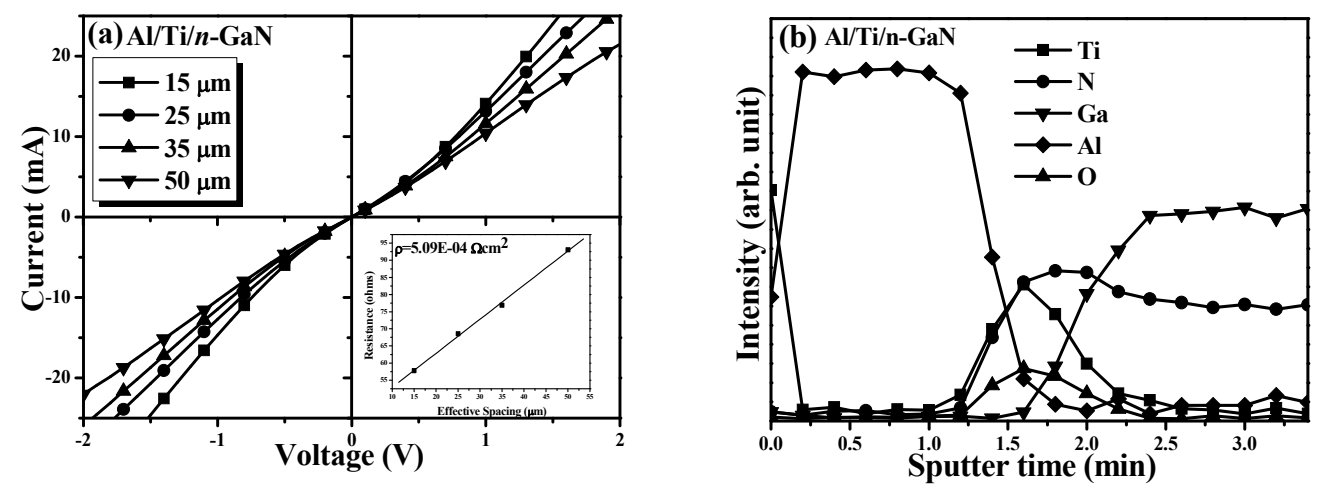

Figure 4. (a) $I-V$ curve of the as-deposited metallic Ti/Al system's contact to $n$-GaN epilayer and (b) the elemental distribution of the $\mathrm{Al} / \mathrm{Ti} / n-\mathrm{GaN}$ contact system conducted from AES depth profile measurement.

The above-mentioned structures' contact to the $n-\mathrm{GaN}$ epilayer demonstrated that the transparent electrodes, such as ITO and cosputtered ITO-ZnO films, have a nonlinear $I-V$ curve even when annealed at an elevated temperature, whereas the opaque $\mathrm{Ti} / \mathrm{Al}$ system had ohmic contact behavior when contacting the $n$-GaN epilayer without a post-annealing treatment. Therefore, we employed a thin Ti interlayer which was deposited between the interface of ITO-ZnO and $n$-GaN to achieve a true ohmic contact system with optical transparency at the visible wavelengths. Figure 5 a shows the $\mathrm{I}-V$ characteristics of the ITO- $\mathrm{ZnO} / \mathrm{Ti} / n-\mathrm{GaN}$ contact systems as a function of the thickness of the Ti interlayer, measured from the contact spacing of $15 \mu \mathrm{m}$ (the $I-V$ curve of the $\mathrm{Al} / \mathrm{Ti} / n-\mathrm{GaN}$ contact system also is shown for comparison). The cosputtered ITO-ZnO film directly contacted the $n$-GaN epilayer, which resulted in a nonlinear $I-V$ property that became an ohmic contact behavior when a thin Ti interlayer was deposited prior to the deposition of the cosputtered ITO-ZnO film. The current flow of the nonalloyed ITO- $\mathrm{ZnO} / \mathrm{Ti} / n-\mathrm{GaN}$ contact system was increased initially as the thickness of the Ti interlayer increased, but it decreased as the thickness of the Ti interlayer reached $10 \mathrm{~nm}$. It was worth noting that the current of the ITO- $\mathrm{ZnO} / n-\mathrm{GaN}$ contact structure inset by an 8-nm-thick Ti interlayer at a bias of $2 \mathrm{~V}$ obviously was higher than that of the metallic Ti/Al structure's contact to the $n-\mathrm{GaN}$ epilayer. The evolution of the specific contact resistance as a function of the Ti interlayered thickness in the ITO-ZnO/Ti $/ n-G a N$ contact system is illustrated in Figure $5 \mathrm{~b}$. The specific contact resistances of these ITO- $\mathrm{ZnO} / \mathrm{Ti} / n-\mathrm{GaN}$ contact systems as well as the $\mathrm{Ti} / \mathrm{Al}$ ohmic contact to the $n$-GaN epilayer are summarized in Table 2. A specific contact resistance of $2.5 \times 10^{-3} \Omega \cdot \mathrm{cm}^{2}$ was obtainable from the cosputtered ITO-ZnO film's contact to the $n$-GaN epilayer with an additional 2-nm-thick Ti interlayer inset between the interface of ITO-ZnO and $n$-GaN. As the thickness of the Ti interlayer reached $8 \mathrm{~nm}$, the specific contact resistance was optimized further to $4.8 \times 10^{-5} \Omega \cdot \mathrm{cm}^{2}$, a value that was a little better than that of the metallic Ti/Al contact to the $n-G a N$ epilayer. The optical transmittances of these $\mathrm{Ti} / \mathrm{ITO}-\mathrm{ZnO}$ systems with various Ti interlayered thicknesses are given in Figure 6 (the optical transmittance of the cosputtered ITO-ZnO film also is shown for comparison). The thin Ti interlayer did not change the onset absorption of the cosputtered ITO-ZnO film at the near-UV wavelength. However, the optical transparency of the Ti/ITO-ZnO systems was reduced gradually as the thickness of the Ti interlayer increased. The average transmittances at the visible wavelengths of these $\mathrm{Ti} / \mathrm{ITO}-\mathrm{ZnO}$ systems are listed in Table 2 . It can be found that the average transmittances of these $\mathrm{Ti} / \mathrm{ITO}-\mathrm{ZnO}$ systems decreased monotonously from $83.2 \%$ to $48.8 \%$ as the thickness of the Ti interlayer was increased from 2 to $10 \mathrm{~nm}$. Accordingly, although the cosputtered ITO-ZnO film's ohmic contact to the $n$-GaN epilayer can be optimized when an 8-nm-thick Ti interlayer is inset between the ITO- $\mathrm{ZnO} / n$ - $\mathrm{GaN}$ interface, the structural transmittance at the visible wavelengths apparently decreased to $63.2 \%$ as compared to the cosputtered ITO-ZnO film $(\sim 84.6 \%)$. Herein, we proposed the figure of merit (FOM), $\Phi$, to quantitatively determine the performance of these 
transparent electrodes' ohmic contact to the $n-G a N$ epilayer. This value is calculated according to the following expression which takes into consideration both the specific contact resistance, $\rho_{\mathrm{C}}$, and the average optical transmittance, $T_{\text {ave }}[19,20]$ :

$$
\Phi=\frac{T^{10}}{\rho_{c}}
$$
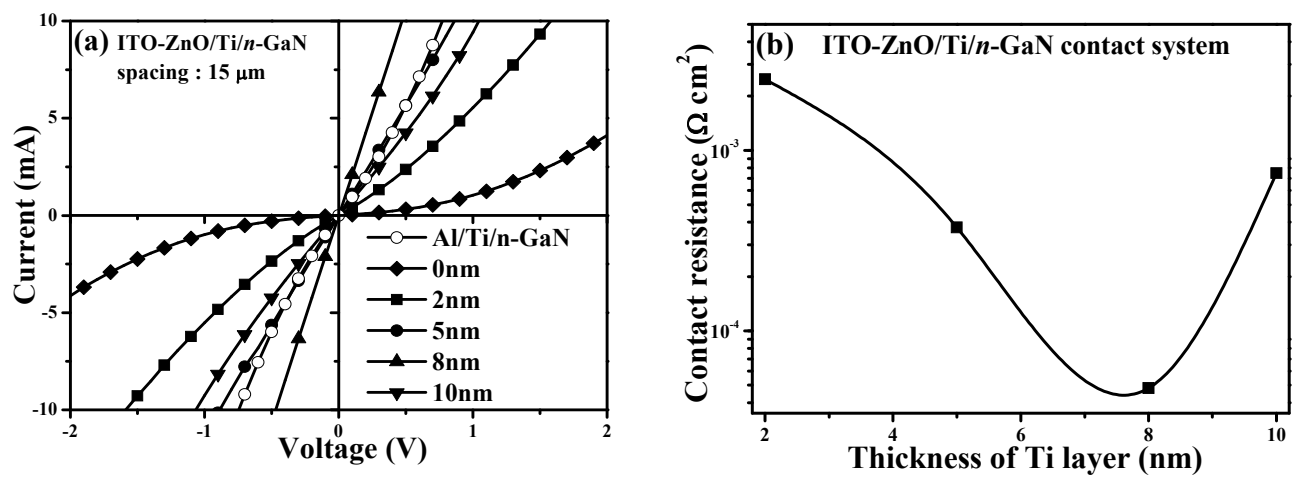

Figure 5. (a) $I-V$ characteristics of the ITO- $\mathrm{ZnO} / \mathrm{Ti} / n-\mathrm{GaN}$ contact system as a function of the $\mathrm{Ti}$ interlayered thickness, measured from the contact spacing of $15 \mu \mathrm{m}$ (the $I-V$ curve of the $\mathrm{Al} / \mathrm{Ti} / n-\mathrm{GaN}$ contact system also is shown for comparison) and (b) the corresponding specific contact resistance as a function of the Ti layered thickness.

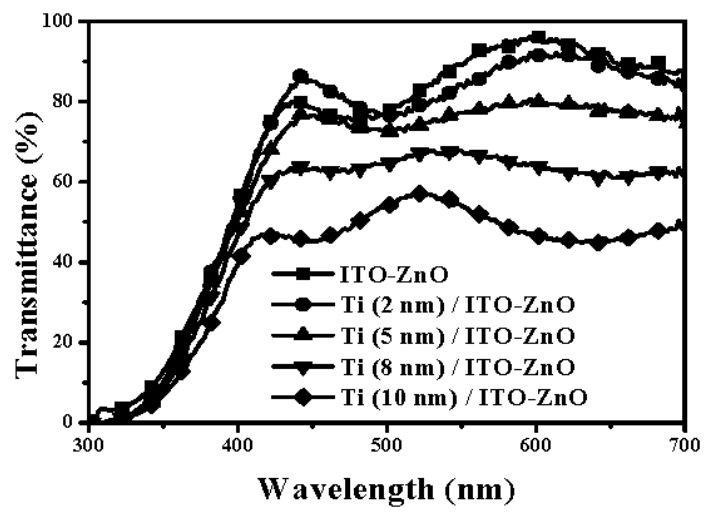

Figure 6. Optical transmittance of the Ti/ITO-ZnO systems as a function of the Ti interlayered thickness (the optical transmittance of the pure ITO-ZnO also is shown for comparison).

Table 2. Specific contact resistance, optical average transmittance, and FOM of the Ti/ITO-ZnO systems as a function of the thickness of the Ti interlayer (the specific contact resistance of the $\mathrm{TI} / \mathrm{Al}$ contact to the $n$-GaN epilayer also is given for comparison).

\begin{tabular}{cccc}
\hline Thickness of Ti Interlayer & $\rho_{\mathbf{c}}\left(\Omega \cdot \mathbf{c m}^{\mathbf{2}}\right)$ & $\boldsymbol{T}_{\text {ave }} \mathbf{( \% )}$ & FOM $\left(\Omega^{-1} \cdot \mathbf{c m}^{-2}\right)$ \\
\hline Ti/Al & $5.1 \times 10^{-5}$ & - & - \\
$0 \mathrm{~nm}$ & - & 84.6 & - \\
$2 \mathrm{~nm}$ & $2.5 \times 10^{-3}$ & 83.2 & 49 \\
$5 \mathrm{~nm}$ & $3.8 \times 10^{-4}$ & 75.1 & 144 \\
$8 \mathrm{~nm}$ & $4.8 \times 10^{-5}$ & 63.2 & 203 \\
$10 \mathrm{~nm}$ & $7.8 \times 10^{-4}$ & 48.8 & $<1$ \\
\hline
\end{tabular}

The higher the FOM of the Ti/ITO-ZnO system obtained, the better the quality of the transparent electrode contacted to the $n$-GaN epilayer. Table 2 gives the FOM of the Ti/ITO-ZnO systems as a 
function of the thickness of the Ti interlayer. A highest FOM of 203 was achieved from the Ti/ITO-ZnO system with an 8-nm-thick Ti interlayer contacting the $n$-GaN epilayer. Moreover, the FOM obviously was reduced to a value lower than unity for the cosputtered ITO- $\mathrm{ZnO}$ film's contact to the $n$-GaN epilayer while inset by a 10-nm-thick Ti interlayer. This was the result of both the specific contact resistance and average optical transmittance being apparently inferior to that of the sample inset by a Ti interlayer with the thickness of $8 \mathrm{~nm}$. Figure $7 \mathrm{a}, \mathrm{b}$ show the elemental distributions of the cosputtered ITO-ZnO film directly contacting the $n$-GaN epilayer and an 8-nm-thick Ti interlayer inset between the interface of ITO-ZnO and $n$-GaN, respectively. The cosputtered ITO-ZnO film's contact to the $n-\mathrm{GaN}$ epilayer had significant interdiffusion at the ITO- $\mathrm{ZnO} / n-\mathrm{GaN}$ interface, especially for the $\mathrm{O}$ and $\mathrm{N}$ atoms. The outdiffusion of the $\mathrm{N}$ atoms at the $n-\mathrm{GaN}$ surface led to an increase in the electron carriers at the $n$-GaN surface, whereas the indiffusion of the $\mathrm{O}$ atoms to the $n$-GaN surface from the ITO-ZnO film was proven to result in the compensation of the carriers and impede the carrier transmission, thereby exhibiting the Schottky property, as shown in Figure 3. In contrast to the cosputtered ITO-ZnO film directly contacting the $n$-GaN epilayer, a thin Ti interlayer inset between the interface of ITO- $\mathrm{ZnO}$ and $n-\mathrm{GaN}$ resulted in more $\mathrm{N}$ atoms being outdiffused from the $n$-GaN surface, as shown in Figure $7 \mathrm{~b}$. In addition, the indiffusion of the $\mathrm{O}$ atoms to the $n$-GaN epilayer, which was harmful to the carriers' transmission, was depressed by the additive Ti interlayer. Accordingly, this nonalloyed ITO-ZnO/Ti/ $n-G a N$ contact system had excellent ohmic contact behavior with optical transmittance.
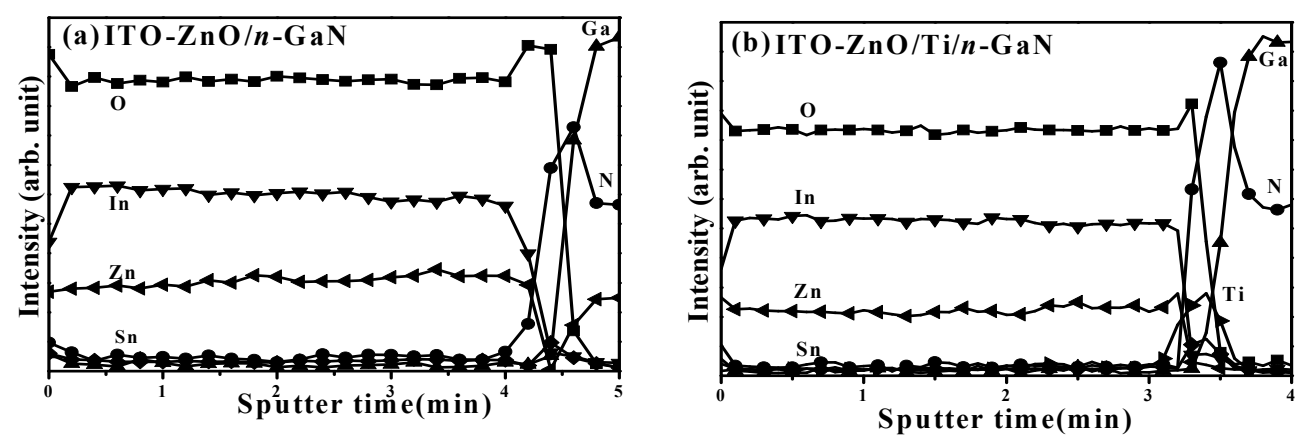

Figure 7. Elemental distributions of (a) the cosputtered ITO-ZnO film directly contacting the $n$-GaN epilayer and (b) the ITO-ZnO/n-GaN contact system inset by an 8-nm-thick Ti layer conducted from AES depth profile measurements.

\section{Conclusions}

Transparent conductive ITO and cosputtered ITO-ZnO films were used as ohmic contact electrodes to an $n$-GaN epilayer. Both of these TCO films had an average transmittance higher than $80 \%$ at the visible wavelengths. However, although the cosputtered ITO-ZnO film had a resistivity $\left(\sim 3.82 \times 10^{-4} \Omega \cdot \mathrm{cm}\right)$ that was almost an order of magnitude lower than that of the ITO film, the obvious indiffusion of the $\mathrm{O}$ atoms into the $n-\mathrm{GaN}$ epilayer was observed from the AES depth profile. This led to the ITO-ZnO/n-GaN contact system having Schottky contact behavior even when processed by an RTA treatment. The nonlinear $I-V$ characteristic of the ITO- $\mathrm{ZnO} / n-\mathrm{GaN}$ contact system evolved into a true ohmic contact when a thin Ti interlayer was introduced between the interface of ITO-ZnO and $n-\mathrm{GaN}$. The specific contact resistance of the ITO-ZnO/Ti/n-GaN contact system initially was decreased with the thickness of the Ti interlayer increasing and then degraded as the inset Ti interlayer reached $10 \mathrm{~nm}$, whereas the optical transmittance at the visible wavelengths monotonously decreased as the interlayer thickness increased. Combined with the specific contact resistance and the average optical transmittance at the visible wavelengths, the highest FOM was obtainable from the ITO- $\mathrm{ZnO} / \mathrm{Ti} / n-\mathrm{GaN}$ contact system with the $8 \mathrm{~nm}$ thickness of the Ti interlayer, which exhibited a specific contact resistance of $4.8 \times 10^{-5} \Omega \cdot \mathrm{cm}^{2}$ and an average optical transmittance 
of $63.2 \%$. This ohmic contact behavior achieved from the nonalloyed ITO-ZnO/Ti/ $n-\mathrm{GaN}$ contact system was achieved by this mechanism: the inset Ti interlayer functioned to effectively induce the outdiffusion of the $\mathrm{N}$ atoms at the $n$-GaN surface and to suppress the indiffusion of the $\mathrm{O}$ atoms from the cosputtered ITO-ZnO film to the $n$-GaN epilayer.

Acknowledgments: This work was supported by Ministry of Science and Technology under Ministry of Science and Technology 103-2221-E-150 -067 and Industrial Technology Research Institute South under FY104-AE2.

Author Contributions: Day-Shan Liu organized and designed the experiment procedures; Tai-Hong Chen, Li-Wen Lai, and Ching-Ting Lee supported the measurement results and wrote this paper; Wei-Hua Hsiao, Jyun-Yong Li, Hong-Jyun Lin, and Nan-Jay Wu executed the film depositions, measurements and resulting structures analysis. All authors read and approved the final version of the manuscript to be submitted.

Conflicts of Interest: The authors declare that there is no conflict of interests regarding the publication of this paper.

\section{References}

1. Ho, C.C.; Lai, L.W.; Lee, C.C.; Yang, K.C.; Lai, B.T.; Liu, D.S. Transparent cosputtered ITO-ZnO electrode ohmic contact to $n$-type $\mathrm{ZnO}$ for $\mathrm{ZnO} / \mathrm{GaN}$ heterojunction light-emitting diode. J. Phys. D: Appl. Phys. 2013, 46. [CrossRef]

2. Wang, M.W.; Gao, B.A.; Lin, D.J.; Chen, J.H. Copper aluminum nano junction normal temperature processes and methods applied to LED high heat transfer performance. Smart Sci. 2014, 2, 36-43.

3. Khanna, R.; Pearton, S.J.; Ren, F.; Kravchenko, I.I. Stability of Ti/Al/ZrB 2 /Ti/Au ohmic contacts on $n$-GaN. Appl. Surf. Sci. 2006, 253, 2340-2344. [CrossRef]

4. Nam, Y.I.; Lee, B.T. Investigation of $\mathrm{Ti} / \mathrm{Au}$ and $\mathrm{Ti}_{2} \mathrm{~N} / \mathrm{Ti} / \mathrm{Au}$ ohmic contacts to $n$-GaN films. Semicond. Sci. Technol. 2011, 26. [CrossRef]

5. Moon, S.Y.; Son, J.H.; Choi, K.J.; Lee, J.L.; Jang, H.W. Indium as an efficient ohmic contact to N-face $n$-GaN of GaN-based vertical light-emitting diodes. Appl. Phys. Lett. 2011, 99, 202106. [CrossRef]

6. Sheu, J.K.; Su, Y.K.; Chi, G.C.; Jou, M.J.; Liu, C.C.; Chang, C.M. Indium tin oxide ohmic contacted to highly doped $n$-GaN. Solid State Electron. 1999, 43, 2081-2084. [CrossRef]

7. Tang, B.T.; Yu, Q.X.; Lee, H.Y.; Lee, C.T. Ohmic perfromance of $\mathrm{ZnO}$ and ITO/ZnO contacted with $n$-type GaN layer. Mater. Sci. Eng. B 2001, 82, 259-261. [CrossRef]

8. Hwang, J.D.; Yang, G.H.; Lin, C.C.; Chang, S.J. Nonalloyed Ti/indium tin oxide and Ti ohmic contacts to n-type GaN using plasma pre-treatment. Solid State Electron. 2006, 50, 297-299. [CrossRef]

9. Liu, S.Y.; Lin, Y.C.; Ye, J.C.; Tu, S.J.; Huang, F.W.; Lee, M.L.; Lai, W.C.; Sheu, J.K. Hydrogen gas generation using $n$-GaN photoelectrodes with immersed Indium Tin Oxide ohmic contacts. Opt. Express 2011, 19, A1196-A1201. [CrossRef] [PubMed]

10. Kang, K.M.; Jo, J.M.; Kwak, J.S.; Kim, H.; Kim, Y.S.; Sone, C.; Park, Y. In/ITO ohmic contacts to Ga-face and $\mathrm{N}$-face $n$-GaN for InGaN-based Light-emitting diodes. J. Korean Phys. Soc. 2009, 55, 318-321.

11. Guo, H.; Brown, K.; Korakakis, D.; Cao, X.A. Low-resistance, highly transparent, and thermally stable Ti/ITO Ohmic contacts to $n$-GaN. J. Vac. Sci. Technol. B 2009, 27, 1161-1164. [CrossRef]

12. Liu, D.S.; Wu, C.C.; Lee, C.T. A transparent and conductive oxide film prepared by RF magnetron co-sputtering system at room temperature. Jpn. J. Appl. Phys. 2005, 44, 5119-5121. [CrossRef]

13. Liu, D.S.; Lin, C.H.; Tsai, F.C.; Wu, C.C. Microstructure investigations of ITO films cosputtered with $\mathrm{ZnO}$ at room temperature. J. Vac. Sci. Technol. A 2006, 24, 694-699. [CrossRef]

14. Moons, E.; Goossens, A.; Savenije, T. Surface photovoltage of porphyrin layers using the kelvin probe technique. J. Phys. Chem. B 1997, 101, 8492-8498. [CrossRef]

15. Sheu, J.K.; Su, Y.K.; Chi, G.C.; Jou, M.J.; Liu, C.C.; Chang, C.M.; Hung, W.C.; Bow, J.S.; Yu, Y.C. Investigation of the mechanism for Ti/Al ohmic contact on etched n-GaN surfaces. J. Vac. Sci. Technol. B 2000, 18, 729-732. [CrossRef]

16. Cao, X.A.; Piao, H.; LeBoeuf, S.F.; Li, J.; Lin, J.Y.; Jiang, H.X. Effects of plasma treatment on the ohmic characteristics of Ti/Al/Ti/Au contacts to $n-\mathrm{AlGaN}$. Appl. Phys. Lett. 2006, 89. [CrossRef]

17. Rajagopal, V.R.; Ramesh, C.K. Low-resistance ohmic contacts to $n$-type $\mathrm{GaN}$ using Ti/Al/Re/Au multilayer scheme. J. Optoelectron. Adv. Mater. 2004, 6, 177-182. 
18. Sun, J.; Feng, Q.; Bian, J.; Yu, D.; Li, M.; Li, C.; Liang, H.; Zhao, J.; Qiu, H.; Du, G.J. Ultraviolet electroluminescence from $\mathrm{ZnO}$-based light-emitting diode with $p$-ZnO:N/n-GaN:Si heterojunction structure. J. Lumin. 2011, 131, 825-828. [CrossRef]

19. Liu, J.; Hains, A.W.; Servaites, J.D.; Ratner, M.A.; Marks, T.J. Highly conductive bilayer transparent conducting oxide thin films for large-area organic photovoltaic cells. Chem. Mater. 2009, 21, 5258-5263. [CrossRef]

20. Hudaya, C.; Park, J.H.; Lee, J.K. Effects of process parameters on sheet resistance uniformity of fluorine-doped tin oxide thin films. Nanoscale Res. Lett. 2012, 7. [CrossRef] [PubMed] article distributed under the terms and conditions of the Creative Commons by Attribution (CC-BY) license (http://creativecommons.org/licenses/by/4.0/). 